\title{
Endogenous glutathione levels modulate the frequency of both spontaneous and long wavelength ultraviolet induced mutations in human cells
}

\author{
L.A.Applegate ${ }^{1}$, D.Lautier, E.Frenk ${ }^{1}$ and R.M.Tyrrell ${ }^{2}$ \\ Swiss Institute for Experimental Cancer Research (ISREC), ch. des \\ Boveresses 155, 1066 Epalinges/Lausanne and 'University Hospital \\ (CHUV), Department of Dermatology, CH-1011 Lausanne, Switzerland \\ ${ }^{2}$ To whom correspondence should be addressed
}

Spontaneous and induced mutations at the hypoxanthine guanine phosphoribosyl transferase locus have been measured in cultured human lymphoblastoid (TK6) cell populations under conditions in which cellular glutathione has been severely depleted by overnight treatment with buthionine-S, $R$ sulfoximine. At maximum levels of glutathione depletion, the increase in spontaneous frequency is at least 5-fold, a finding consistent with the possibility that cellular redox state can modulate the levels of pre-mutagenic damage arising as a result of normal metabolism in cultured human cells. Glutathione depletion does not lead to a significant enhancement in the frequency of mutants that arise as a result of irradiation at $313 \mathrm{~nm}$ but does lead to a 3-fold increase in mutations resulting from irradiation at $365 \mathrm{~nm}$. These results indicate that glutathione may quench reactive intermediates that would otherwise lead to spontaneous mutations as well as a fraction of UVA radiation-induced premutagenic damage.

\section{Introduction}

The appearance of mutations at specific genetic loci remains the most powerful indicator that a particular set of conditions pose a genetic hazard and that such conditions will lead to an enhanced cancer risk when extrapolated to complex multicellular organisms. Thus the highly genotoxic and mutagenic UVB $(290-320 \mathrm{~nm})$ component of sunlight is held to be largely responsible for the carcinogenic action of natural sunlight which is the agent leading to the vast majority of human skin cancer (1). The UVA $(320-380 \mathrm{~nm})$ component of sunlight is also known to be carcinogenic (2) and to cause genetic damage (3) but the results of mutation studies with cultured mammalian cells are less clear (4). Reports of mutation induction by broad-band sources fail to exclude the contribution of shorter wavelengths and results using monochromatic radiation at various UVA wavelengths vary with the wavelength and cell type employed (5-7). For example the results of our own study (5) did not demonstrate mutation induction at the hypoxanthine guanine phosphoribosyl transferase (HGPRT ${ }^{*}$ ) locus by radiation at a wavelength of $365 \mathrm{~nm}$ in cultured human lymphoblastoid cells whereas such radiation does appear to be mutagenic in a human teratocarcinoma cell line (6) and in cultured human fibroblasts (7).

At least part of the cell type variability in susceptibility to UVA mutagenesis may be related to observations that UVA radiation

\footnotetext{
*Abbrevlations: HGPRT, hypoxanthine guanine phosphoribosyl transferase; $\mathrm{TG}^{\mathrm{r}}$, thioguanine resistant; BSO, D,L-buthionine- $S, R$-sulfoximine; TCA, trichloroacetic acid; GSH and GSSG, reduced glutathione and its oxidized form (glutathione will be written in full where the two forms are not distinguished analytically); NEM, N-ethylmaleimide.
}

constitutes an oxidative stress to cells (8). Subtle variations in cellular antioxidant defence pathways could have a major influence on the levels of damage induced, including premutagenic damage. Clearly, cellular redox state and in particular, glutathione levels would be expected to influence the susceptibility of cells to oxidant mutagens. Endogenous glutathione provides a major constitutive defence against the cytotoxic action of both UVB and UVA radiations $(9,10)$. Furthermore the induction of heme oxygenase, which appears to be a general response to oxidant stress including UVA radiation (11), is also strongly influenced by endogenous glutathione levels (12). In view of these findings we have examined the influence of cellular glutathione levels on the frequency of mutants that arise as a result of irradiation of cultured human cells with specific wavelengths in the solar UV range. These studies have led us to the observation that the rate of appearance of mutations that arise spontaneously can be dramatically increased by the depletion of intracellular glutathione.

\section{Materials and methods}

\section{Cell culture}

The TK6 human lymphoblastord cell line onginally selected by Thilly (13) has a high cloning efficiency $(80-100 \%)$ on plastic microtiter plates. TK6 populations were routinely grown in RPMI medium (Seromed) supplemented with $10 \%$ fetal calf serum, glutamune, sodium bicarbonate, penicillin and streptomycin (complete medrum). Cells were grown at $37^{\circ} \mathrm{C}$ in a $5 \% \mathrm{CO}_{2}$ incubator. Under these conditions the doubling time is $18-20 \mathrm{~h}$. Culures were mantained in exponential growth by diluting to $2 \times 10^{5}$ cells $/ \mathrm{ml}$ every 2 or 3 days.

Lymphoblastoid cell populations were purged of thioguanine resistant $\left(\mathrm{TG}^{2}\right)$ mutants by growing in complete medium containing $10^{-5} \mathrm{M}$ deoxycytidine, $2 \times 10^{-4} \mathrm{M}$ hypoxanthine, $1.75 \times 10^{-5} \mathrm{M}$ thymidine and $2 \times 10^{-7} \mathrm{M}$ aminopterin for 3 days. The medium was then changed to a similar one containung all of the additives except amnopterin. After $24 \mathrm{~h}$ the cells were changed to complete medium (no addituves) for a final $24 \mathrm{~h}$ incubation. Such cell populations were then alıquoted and frozen in liquid nitrogen for use in starter cultures throughout the study.

Irradiation conditions

Cell suspensions were irradiated in quartz vessels with mochanical stirring at a temperature between 2 and $5^{\circ} \mathrm{C}$. All irradiations were performed under yellow light. Monochromatic radiation was obtained from a single GM 250 Schoefel monochromator equipped with a $2.5 \mathrm{~kW}$ Mercury-Xenon lamp (Hanovia). Halfband widths were 8 and $17.6 \mathrm{~nm}$ for 313 and $365 \mathrm{~nm}$ respectively. In addition, Corning filters $0-54(0.5 \mathrm{~mm})$ were employed for $313 \mathrm{~nm}$ and $0-52(2 \mathrm{~mm})$ were employed for $365 \mathrm{~nm}$. Radiation fluences were monitored by an International Light radiometer (IL 1700) for esch experiment.

Glutathione depletion

Cell cultures were depleted of glutathione by the addition of various concentrations of $\mathrm{D}, \mathrm{t}$-buthionine- $S, R$-sulfoximine (BSO, an inhibitor of $\gamma$-glutamyl-cysteine synthetase) to complete medium $18 \mathrm{~h}$ prior to experimentation.

Cloning assay

TK6 cell suspensions were counted electronically and diluted in complete medium to a concentration compatible with the inoculation of two cells per well in a volume of $200 \mu \mathrm{l}$ in 96-well microtiter plates (Costar). After 14-21 days, clones were scored microscopically and the cloning efficiency determined using the equation:

$$
\text { cloning efficiency }=-\ln P_{0} / n
$$

where $P_{0}=$ fracton of wells without clones, and $n=$ average number of cells originally placed per well. UV irradiated and BSO-treated cultures were diluted for cloning in order to compare surviving fractions with those of the control. 
Incubations of cell cultures for $18 \mathrm{~h}$ with up to $500 \mu \mathrm{M}$ BSO resulted in negligible toxicity to control (unirradiated) cultures.

\section{Glutathione measurements}

Following various treatments, TK6 cells were pelleted by centrifugation and rinsed twice with cold PBS and then counted prior to centrifuging at $4^{\circ} \mathrm{C}$ and resuspended in cold PBS. Cellular extractions for glutathione were performed with freshly prepared 5\% trichloroacetic acid (TCA) in 2 mM EDTA at a concentration of $2 \times 10^{6} \mathrm{cells} / \mathrm{ml}$. Following centrifugation at $4^{\circ} \mathrm{C}$, each supernatant was divided in talf to permit measurement of both total intracellular glutathione and its oxidized form, glutathione disulfide (GSSG), using a spectrophotometric method adapted from Tietze (13). In general, $N$-ethylmaleimide (NEM) was added to a final concentration of $50 \mathrm{mM}$ and the samples incubated for $1 \mathrm{~h}$ at $25^{\circ} \mathrm{C}$ for GSSG determination. Removal of NEM was accomplished by 10 ether extractions prior to the GSSG rectuctase recycling assay using 5,5'-dithio-bis(-2-nitrobenzoic acid) (DNTB). Data were then expressed as the percentage of total intracellular glutathione equivalents (GSH +2 GSSG) and are the means of 2-10 independent experiments.

Mutarion assay

After glutathione depletion and/or UV irradiation, cell populations were resuspended in complete medium at $3 \times 10^{5} \mathrm{cells} / \mathrm{ml}$. Aliquots from each experimental condition were plated for survival determination. Treated TK6 cells were maintained under exponential growth for 10 days for complete expression of thioguanine resistance and then distributed in an appropriate number of sets of $5 \times 96$-well plates $\left(4 \times 10^{4}\right.$ cells/well) containing $3 \times 10^{-5} \mathrm{M}$ thioguanine. After 14-21 days, clonal growth was scored microscopically and mutant frequencies were calculated using the cloning efficiency observed in non-selective medium.

\section{Results}

Modulation of endogenous glutathione levels by incubation with BSO

BSO is a highly specific inhibitor of $\gamma$-glutamyl-cysteine synthetase (15) which causes the depletion of cellular glutathione during an extended incubation under normal growth conditions. Using a standard incubation time of $18 \mathrm{~h}$, human lymphoblastoid cell populations (TK6) may be progressively depleted of both reduced glutathione (GSH) and its oxidized form (GSSG) by using a range of BSO concentrations (Figure 1). Maximum depletion requires a BSO concentration in excess of $100 \mu \mathrm{M}$, a 10-fold higher concentration than that previously observed to be required for maximum depletion in cultured human skin fibroblasts (10). Furthermore, whereas the levels of glutathione remain low (maximum 20\% of normal values) for several days after BSO exposure in human fibroblast populations (this laboratory, unpublished results), the intracellular concentration of glutathione returns to essentially normal values within $24 \mathrm{~h}$ of removal of the inhibitor from cultured lymphoblastoid populations even after BSO concentrations as high as $50 \mu \mathrm{M}$ (Figure 2). Total intracellular glutathione (GSH + GSSG) in untreated TK6 cells is $5.70 \pm 0.14 \mathrm{nmol}$ GSH equivalents $/ 10^{6}$ cells of which $4 \%$ is GSSG. Under these culture conditions, the extracellular concentration of glutathione is $-65 \%$ of the intracellular value.

The spontaneous mutant frequency is enhanced by glutathione depletion

Under control conditions, the frequency of the occurrence of mutants at the HGPRT locus (spontaneous frequency) was determined to be $3.78 \times 10^{-6} \pm 1.23 \times 10^{-6}$. This is close to the value of $3.67 \times 10^{-6} \pm 2.16 \times 10^{-6}$ obtained from a series of independent determinations in a previous study (5). However, the frequency of spontaneous mutants rises significantly as a result of glutathione depletion (Figure 3). At maximum levels of glutathione depletion ( $>100 \mu \mathrm{M}$ BSO) the increase in frequency is at least 5-fold. Although this observation is of considerable interest, it imposes limitations on the study of induced mutation under conditions of glutathione depletion since the background mutant frequencies are extremely high.

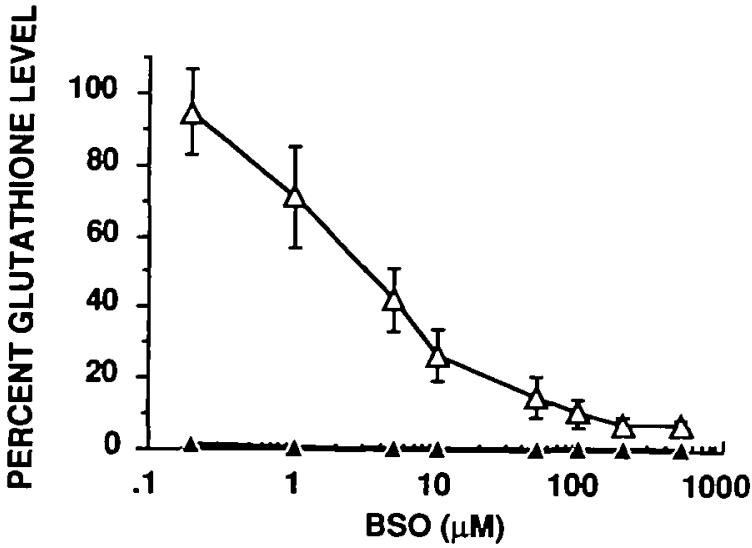

Fig. 1. Percentage reduction in glutathione levels of TK6 cell populations as a function of an $18 \mathrm{~h}$ incubation in various concentrations of BSO. Levels of intracellular glutathione $(\Delta)$ and intracellular GSSG $(\Delta)$ are the average of six to nine determinations shown together with the associated standard deviation.

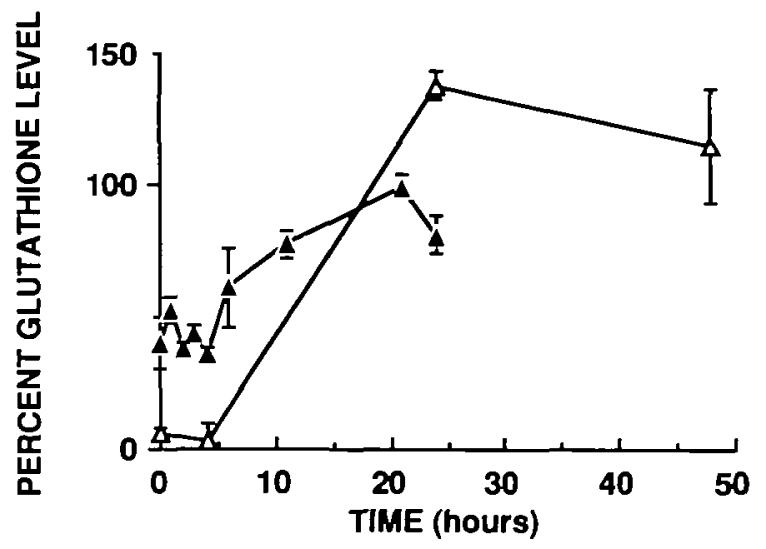

Fig. 2. Recovery of intracellular glutathione as a function of incubation time following $18 \mathrm{~h}$ exposure to either $3 \mu \mathrm{M}(\Delta)$ or $50 \mu \mathrm{M}(\triangle)$ BSO. Values are the average of two to five determinations.

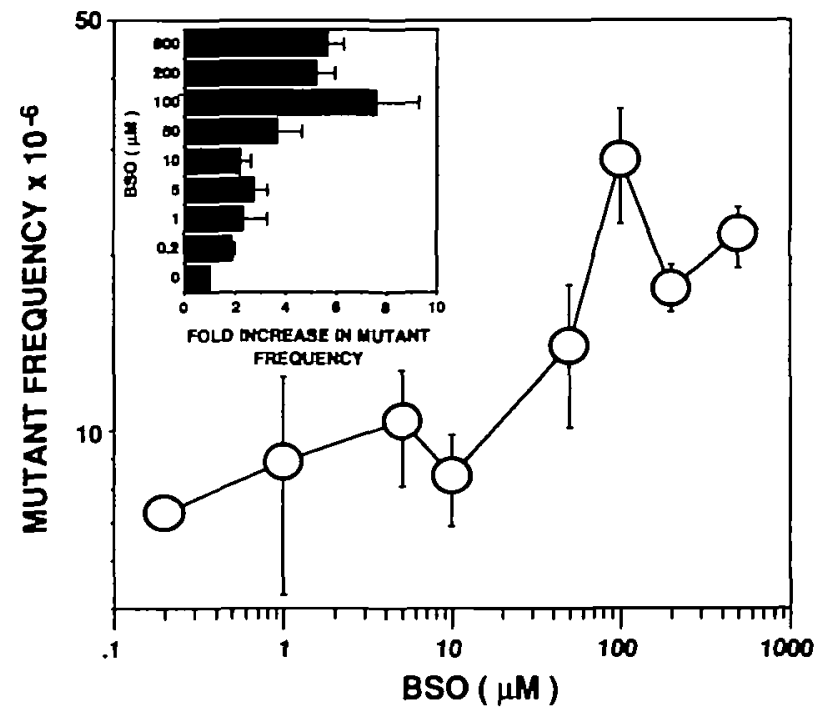

Fig. 3. Frequency of spontaneous mutation at the HGPRT locus of TK6 cell populations as a function of preincubation with a series of concentrations of BSO. Each data point is the mean of 3-13 determinations (five microtiter plates per set) pooled from two independent experiments and shown with the associated standard deviation. The histogram inset shows the fold increase in mutation frequency over the value obtained with no BSO exposure. 
Table I. Influence of glutathione depletion on the frequency of mutants induced at the HGPRT locus by UVA and UVB radiations

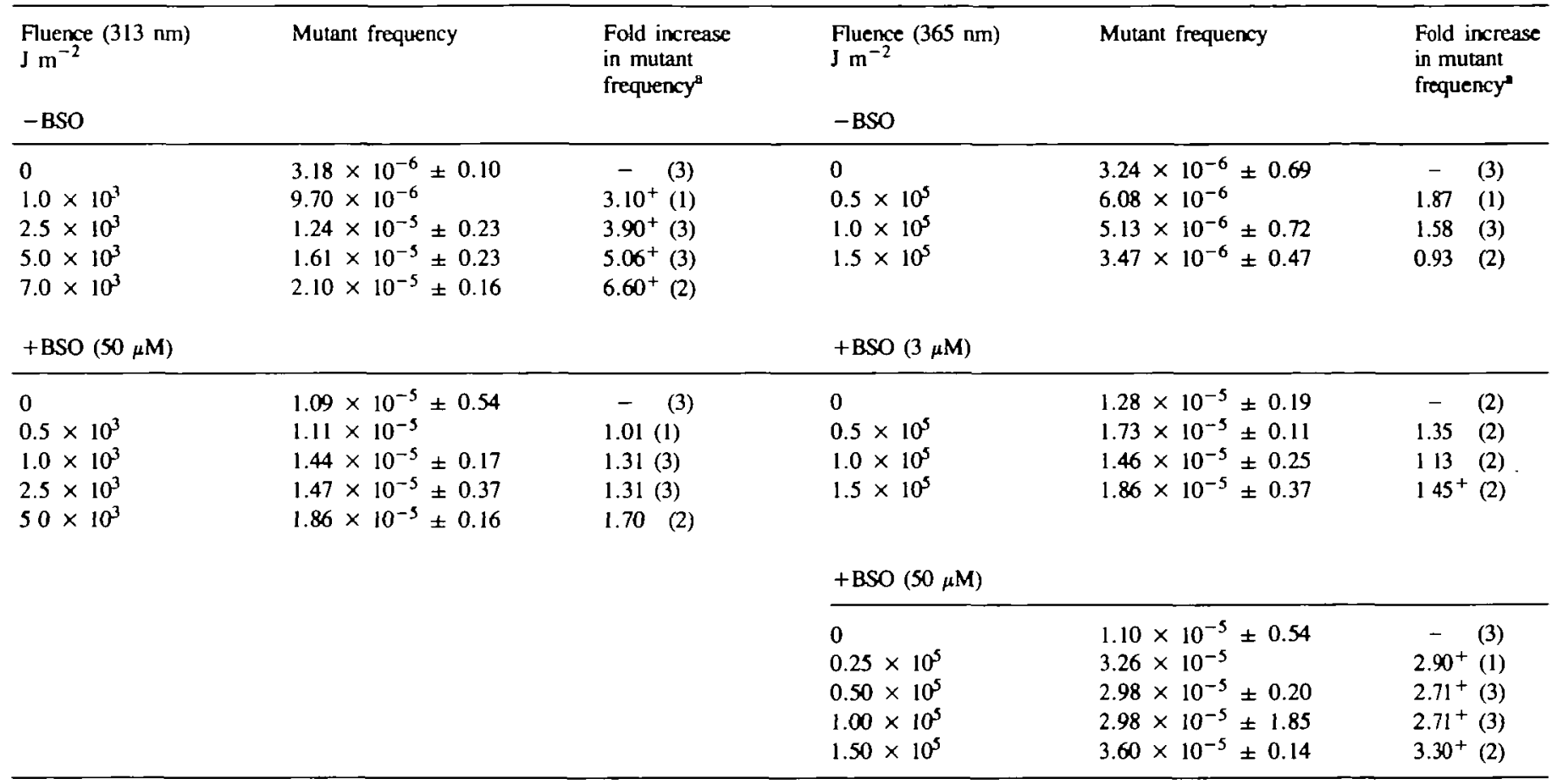

"Figures in parentheses show the number of independent experiments. The superscript ${ }^{+}$is used to indicate that this increase is significant $(P<0.05)$ as assessed by pooling the data from independent experiments (at least five plates per experiment).

Mutagenic action of UVB $(313 \mathrm{~nm})$ and $U V A(365 \mathrm{~nm})$ radiations under conditions of glutathione depletion

Irradiation of TK6 populations at a wavelength of $313 \mathrm{~nm}$ leads to a fluence-dependent increase in the frequency of mutants at the HGPRT locus (Table I, see also ref. 5). Although glutathione depletion sensitizes such cell populations to the lethal action of radiation at this wavelength (Figure 4A) as previously observed for cultured skin fibroblasts (9), the slight increase in mutant frequency observed after thiol depletion is not significant. In contrast, although we have confirmed our previous observation (5) that radiation at $365 \mathrm{~nm}$ does not cause a detectable increase in mutant frequency in TK6 populations over the fluence range employed (Table I), the sensitization of such cell populations to radiation at $365 \mathrm{~nm}$ by glutathione depletion (Figure 4B) is accompanied by a marked increase in mutant frequency (Table I). The -3 -fold enhancement in the frequency of UVA radiationinduced mutants is observed at the lowest fluence $\left(2.5 \times 10^{4} \mathrm{~J}\right.$ $\mathrm{m}^{-2}$ ) employed and no further increases are observed at higher fluences.

\section{Discussion}

A major finding of the present study is that rates of spontaneous mutagenesis at the HGPRT locus of human lymphoblastoid cell populations can be enhanced several-fold by depletion of intracellular glutathione. The concentrations of inhibitor (BSO) which give intermediate levels of enhancement (Figure 3) lie in the same range as those that lead to intermediate levels of glutathione depletion (Figure 1). These results strongly indicate that cellular redox state can modulate the levels of pre-mutagenic damage arising as a result of normal metabolism in cultured human cells. Indeed, these results lend strong support to the argument that spontaneous mutations arise as a result of active

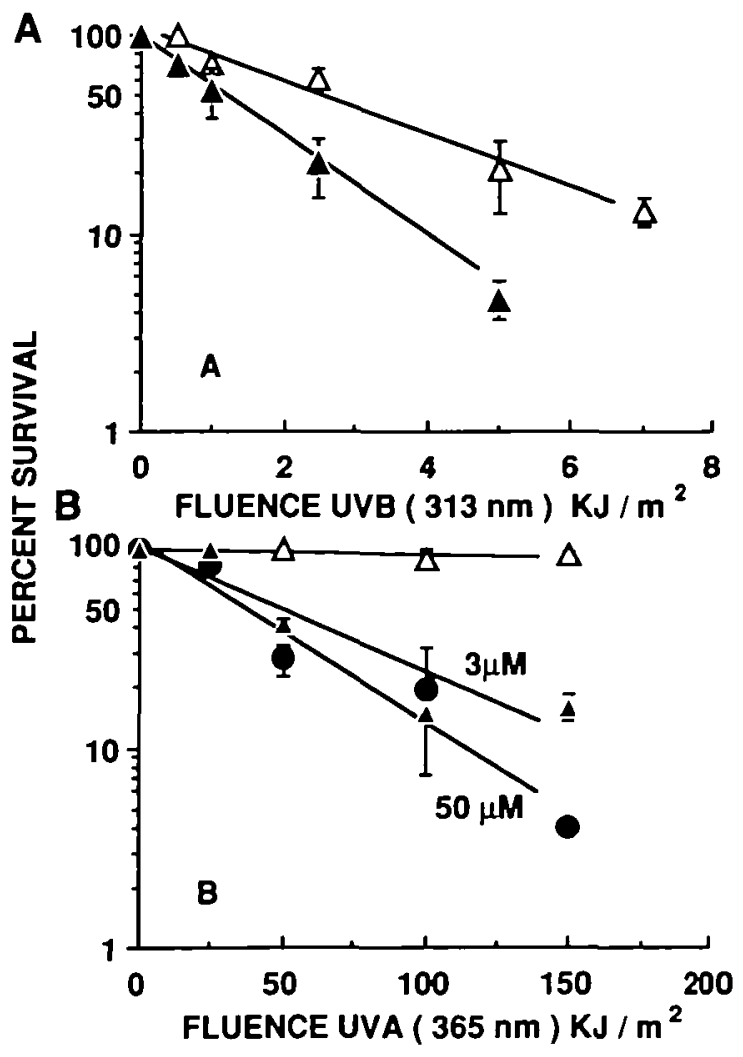

Fig. 4. Survival of TK6 lymphoblastoid cell population as a function of fluence of A, UVB $(313 \mathrm{~nm})$ radiation and B, UVA $(365 \mathrm{~nm})$ radistion with and without BSO pre-treatment ( $\triangle$, no BSO pre-treatment; $\bullet, 3 \mu \mathrm{M}$ BSO; $\triangle, 50 \mu \mathrm{M}$ BSO). The curves are derived from linear regression analysis. 
species generated intracellularly and that an important role of cellular antioxidant defence systems is to protect against such damage. In view of this, it might be expected that humans with severe glutathione deficiencies would be lacking in cellular defence against oxidant damge and would therefore be cancer prone. That the latter has not been reported to be the case is probably due to the fact that glutathione deficiency in humans is normally due to a defect in glutathione synthetase. The accumulation of the precursor $\gamma$-glutamyl-cysteine (eventually broken down to 5-oxoloproline to give the acidosis characteristic of 5-oxoloprolinuria) can presumably at least partially substitute for the scavenging potential normally provided by glutathione. The lack of suitable mutants in glutathione metabolism also means that we cannot exclude the possibility that the enhanced frequency of spontaneous mutants observed in populations depleted of glutathione by BSO treatment is due to a different (and as yet unknown) property of BSO that can affect the equilibrium of mutants in the population or even that BSO is a mutagen itself.

Mutations at the HGPRT locus can arise from a variety of events ranging from point mutations to gross chromosomal rearrangements. The spontaneous mutant frequency at this recessive genetic locus is therefore high and the enhancement by glutathione depletion presumably reflects the diversity of genetic events that can arise in the presence of persistent active intermediates generated by metabolism. On the other hand, mutants at the dominant $\mathrm{Na}^{+} \mathrm{K}^{+}$ATPase locus (normally scored by ouabain resistance) arise almost exclusively as a result of point mutations and are not observed after treatment with nonspecific damaging agents such as ionizing radiation (16). It is therefore noteworthy that glutathione depletion was also unable to modify the level of mutation at this locus (results not shown), lending support to the notion that spontaneous genetic changes occur as a result of a wide range of damage.

Although glutathione depletion does lead to the enhanced cytotoxicity of UVB radiation (Figure 4A, see also ref. 5) such conditions do not lead to a significant enhancement in the frequency of mutants that arise as a result of irradiation at this wavelength (Table I). In contrast, thiol depletion leads to the generation of significant levels of additional HGPRT mutants when the treated cells are irradiated with normally sub-lethal fluences of UVA radiation (Table I). The 3-fold increase over the spontaneous rate observed under conditions of glutathione depletion by $50 \mu \mathrm{M}$ BSO appears to be maximal even at the lowest fluence $\left(2.5 \times 10^{4} \mathrm{~J} \mathrm{~m}^{-2}\right)$ of UVA radiation employed. This lack of fluence response may be related to the observation that, in our hands (see also ref. 5), a value of $3 \times 10^{-5}$ mutants per survivor is close to the maximum mutant frequency observed at the HPGRT locus after UV treatment in this TK6 lymphoblastoid mutation system. Although the result is consistent with the possibility that lowering of cellular redox capacity increases the level of persistent active intermediates generated by UVA radiation to a level which can cause pre-mutagenic damage, conclusive proof would require experiments in a system in which a fluence response can be established and in which expression of glutathione and its precursors can be modulated genetically.

Once BSO has been removed from the cell cultures, glutathione levels appear to recover to normal within at most $24 \mathrm{~h}$ in these human lymphoblastoid cell populations (Figure 2). Clearly this is the window of time in which enhancement of UVA damage may occur. Since conditions of thiol deprivation begin to occur soon after addition of the inhibitor (BSO), spontaneous mutants that arise as a result of lowered glutathione levels may have arisen at any time up to $40 \mathrm{~h}$ following addition of the drug.

In summary, BSO treatment leads to the enhancement of the frequency of spontaneous mutants that arise at the HGPRT locus, presumably as a result of thiol depletion. Furthermore, although UVA radiation does not normally lead to detectable mutations in this particular human cell line, BSO treatment prior to irradiation leads to a significant increase in mutant frequency relative to unirradiated cell populations. Reactive intermediates, normally quenched out by glutathione, appear to be partly responsible for both the mutations that arise spontaneously and possibly those that are generated by treatment with UVA radiation.

\section{Acknowledgements}

The work reported in this paper was supported by grants from the Swiss League Against Cancer and the Swiss National Science Foundation (3.186.088 and 31.30880.91) and was undertaken during the tenure of a Research Training fellowship to L.A.A. by the International Agency for Research on Cancer.

\section{References}

1. Epstein,J.H. (1989) Photomedicine. In Smith,K.C. (ed.) The Science of Photobiology. 2nd edn, Plenum Press, New York, pp. 155-192.

2. Van Weelden,H., de Gruijl,F.R., Van der Putte,J.C., Toonstra,J. and Van der Leun,J.C. (1988) UVA-induced tumours in pigmented hairless mice and the carcinogenic risks of tanning with UVA. Arch. Dermatol. Res., 280, 300-307.

3. Tyrrell,R.M. (1984) Damage and repair from non-ionizing radiations. In Hurst, A. and Nasim,A. (eds), Repairable Lesions in Microorganisms. Academic Press, New York, pp. 85-124.

4. Tyrrell,R.M. and Keyse,S.M. (1990) New trends in photobiology (invited review) the interaction of UVA radiation with cultured cells $J$. Phorachem. Photobiol. B. Biol., 4, 349-361.

5. Tyrrell,R.M. (1984) Mutagenic action of monochromatic radiation in the solar range on human cells. Mutat. Res., 129, 103-110.

6. Jones,C.A., Huberman,E., Cunningham,M.L. and Peak,J.J. (1987) Mutagenesis and cytotoxicity in human epithelial cells by far- and nearultraviolet radiations: action spectra. Radiat. Res., 110, 244-254.

7. Enninga,I.C., Groenendijk,R.T.L., Filon,A.R., Van Zeeland,A.A. and Simons,J.W.I.M. (1987) The wavelength dependence of UV induced pyrimidine dimer formation, cell killing and mutation induction in human diploid skin fibroblasts. Carcinogenesis, 7, 1829-1836.

8. Tyrrell,R.M. (1991) UVA (320-380 nm) radiation as an oxidative stress. In Sies,H. (ed.), Oxidative Stress: Oxidants and Antioxidants. Academic Press, London, pp. 57-83.

9. Tyrrell,R.M. and Pidoux,M. (1986) Endogenous glutathione protects human skin fibroblasts against the cytotoxic action of UVB, UVA and near-visible radiations. Photochem. Photobiol., 44, 561-564.

10. Tyrrell,R.M. and Pidoux,M. (1988) Correlation between endogenous glutathione content and sensitivity of cultured human skin cells to radiation at defined wavelengths in the solar UV range. Photochem. Photobiol., 47, $405-412$.

11. Applegate,L.A., Lischer,P. and Tyrrell,R.M. (1991) Induction of heme oxygenase: a general response to oxidant stress in cultured mammalian cells. Cancer Res. , 51, 974-978.

12. Lautier,D., Lüscher,P. and Tyrrell,R.M. (1992) Endogenous glutathione levels modulate both constitutive and UVA radiation/oxidant-inducible expression of the human heme oxygenase gene. Carcinogenesis, 13, 227-232.

13. Thilly,W.G. (1979) Study of mutagenesis in diploid human fibroblasts. In Hsie,A.W., O'Neill,J.P. and McElheny,Y.K. (eds), Banbury Report 2, Mammalian Cell Mutagenesis: The Maturation of Test Systems. Cold Spring Harbor Laboratory Press, Cold Spring Harbor, pp. 341-350.

14. Tietze,F. (1969) Enzymic methods for quantitative determination of nanogram amounts of total and oxidised glutathione: applications to mammalian blood and other tissues. Anal. Biochem., 27, 502-521.

15. Griffith,D.W., Anderson, M.E. and Meister,A. (1979) Inhibition of glutathione biosynthesis by prothionine sulfoximine ( $S$ n-propyl homocysteine sulfoximine), a selective inhibrtor of $\gamma$-glutamyl cysteine synthetase. $J$. Biol. Chem., 254, $1205-1210$.

16. Arlett,C.F., Tumbull,D., Harcourt,S.A., Lehmann,A.R. and Colella,C.M. (1975) A comparison of the 8-azaguanine and ouabain-resistance systems for the selection of induced mutant chinese hamster cells. Mutat. Res., 33, $261-278$.

Received on March 5, 1992; revised on May 26, 1992; accepted on May 28, 1992 